\title{
A Comparative Study of Different Modulations for Visible Light Communications
}

\author{
Rofhiwa E. NETSIANDA, K. Ouahada and R. Ndjiongue
}

\begin{abstract}
Wireless technology is quickly developing, and is playing an increasing role in the lives of people throughout the world. Radio frequency communication is the current dominating limited wireless technology that is used in communication industry. Visible Light Communication (VLC) is developing and promising to overcome problems faced in RF communications due to its advantages such as license free and unlimited bandwidth .This project focuses on comparing different modulation schemes for VLC technology, which chosen to be Frequency Shift Keying (FSK) and On-Off Keying (OOK). The physical design for both modulations had to be conducted to perform the comparison. The design involved the receiver and the transmitter circuits. The main properties to be analyzed are the cost, bit error rate and the distance to see which modulation performs better. Due to many components used in FSK modulation, more money was spent compared to OOK modulation. Additionally, OOK modulation outperformed the FSK modulation in terms of the curve of bit error rate versus the distance as shown in this paper.
\end{abstract}

Keywords- Visible light communication; On-Off keying (OOK); Frequency shift keying (FSK).

\section{Introduction}

Wireless technology is quickly developing, and is playing an increasing role in the lives of people throughout the world. Radio frequency communication is the current dominating limited wireless technology that is used in communication industry. Due to its limitations, the world is experiencing a growing of wireless usage demand for the current wireless communication systems. Visible Light Communication (VLC) is developing and promising to overcome problems faced in RF communications.

VLC is a shot range wireless communication that uses light emitting diodes (LEDs) for communication and illumination purpose [1]. This communication technology uses the visible light as the source for information transmission.

Rofhiwa E. NETSIANDA

University of Johannesburg

South Africa

Khmaies Ouahada

University of Johannesburg

South Africa

Richard Ndjiongue

University of Johannesburg

South Africa
The advantage of using VLC over the popular RF communication is that VLC uses frequencies other than radio and they unrestricted and license free [1]. VLC is a shot range wireless communication that uses light emitting diodes (LEDs) for communication and illumination purpose [1]. This communication technology uses the visible light as the source for information transmission. The advantage of using VLC over the popular RF communication is that VLC uses frequencies other than radio and they unrestricted and license free [1].

Additionally VLC does not have health risks unlike existing wireless communication. VLC technology is bringing double functions over the existing communication technology RF. It provides communication (data transfer) and illumination, hence has reduced power consumptions [2].

However, this communication has some drawbacks against existing RF communication that is light of VLC cannot penetrate solid objects [3].

In VLC, communication takes place through modulation of the intensity of LED light. The modulation is conducted in a way that light is undetectable to a human eye [4]. There are types of modulation techniques present in VLC communication. These modulations techniques are classified as single-carrier, subcarrier or multi-carrier modulations. The following modulation techniques are used to transmit data over the visible light spectrum and are On-Off keying, Frequency shift keying (FSK), Pulse width Modulation (PWM), Pulse position modulation, Spread frequency shift keying(S-FSK), colour shift keying(CSK) and orthogonal frequency division multiplex (OFDM) [1]

In VLC communication, the selection of the modulation technique is a significant factor to consider. The reason is that application of the modulation in VLC communication determines the performance of it. There are numbers of parameters that influences the choice of a modulation technique and this will be discussed later in this paper. This paper will concentrate in comparing different modulation schemes and this will involve design, implementation as well as conducting tests to see the performance. This implies that after investigating these different modulation schemes, only two modulation schemes will be compared applied in a transceiver circuit.

In VLC as it was stated earlier in the previous section, communication takes place through modulation of the intensity of LED light. The modulation to be conducted in the 
VLC communication, the transceiver circuit must be available. Therefore, the problem of this project lies in designing and implementing an optimum transceiver circuit for a VLC communication. The main area of the problem lies in selection of optimum modulation scheme for the data transmission in VLC communication. The latter can be achieved by comparing the performance of the modulation schemes by studying and examining their properties. The throughput, received signal quality, required channel capacity, complexity and cost are the parameters that make the modulation scheme selection to be the main problem [5]. In this paper, we place our attention and proposal of designing a transmitter and a receiver based on VLC technology and discuss the effect of noise and distance on each modulation scheme implemented in this paper.

The rest of this paper is organized as follows. In Section II, we present the communication system used in the design. Section III discusses the obtained results and the conclusion is given in Section IV.

\section{Communication system description}

VLC is a short range optical wireless communication which uses LED as an optical channel for communication and illumination purpose. This communication technology uses visible spectrum, which ranges between $380 \mathrm{~nm}-780 \mathrm{~nm}$. These wavelengths correspond to the frequencies ranging from $384 \mathrm{THz}$ to $789 \mathrm{THz}$. In VLC technology, LEDs are recommended to be used due to the fast switching characteristics that they have. Other than that, LEDs have benefits such as long lifetime, low power dissipation and high luminous efficient [6].Moreover, LEDs are recommended in VLC technology because their current intensities can be modulated easily [7]. The intensity of the light is modulated in such a way that is undetectable to the human eye, that's illumination functionality have no negative effect [4].

Figure 3 shows the main design of the project. This design is for both OOK and FSK modulations. It can be seen in the diagram that the design is composed of three main parts; that is a transmitter, receiver and the channel. The power supply also plays a role on this project, since is responsible for activating the LED because the arduino cannot provide enough power to LED.

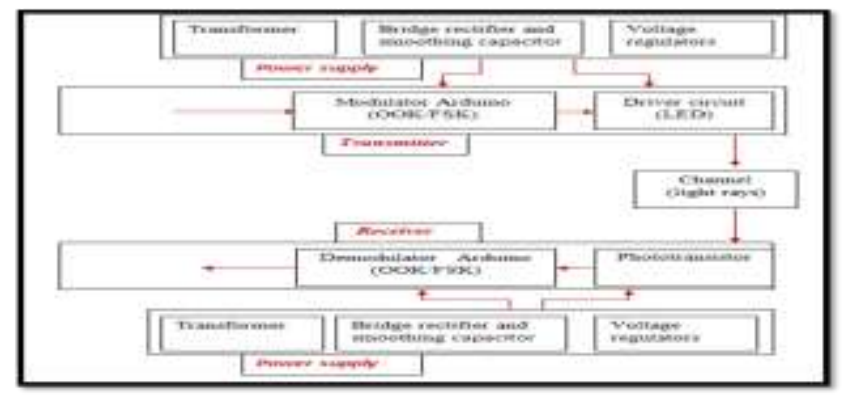

Figure 3: VLC communication system for OOK/FSK
Figure 4 depicts the final design implemented for this project. The design shows the transmitter and the receiver circuit for both OOK and FSK modulation. The transmitter circuit is composed of two LED driver circuits, where one is for OOK and other is for FSK modulation.

The reason of putting two modulations in one Vero board was for minimizing the cost and avoiding having many separated circuits. The receiver circuit is also composed of two demodulations, which are OOK and FSK. The LED of OOK and FSK are place on the same level for the testing purpose.

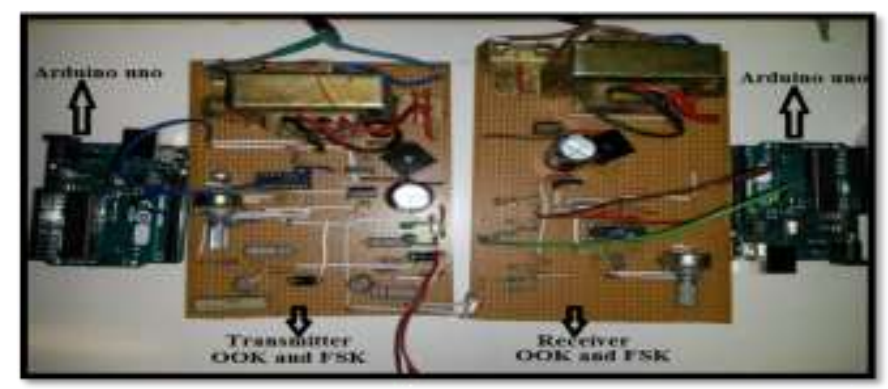

Figure 4: final design of VLC communication transceiver circuits

The design above was discussed in detailed using block diagrams and the circuit. The usage of the block diagram was to give the clear understanding on how the design operates. The design was for both OOK and FSK modulations. It was emphasized that the transmitter circuit included both OOK and FSK in one Vero board and receiver circuit included both too. Having the design implemented, the next chapter will be focusing on the experimental designs needed to test the performance. The experimental design will be break down into sections, each experiment with aim, procedures, apparatus, setup and expected results.

\section{Results and discussion}

In this section, we report and analyze the obtained results from our proposed and designed circuit for our home security VLC. The results shown below illustrate the transmitter and receiver functionality test results, the distance test results, angle test results, and the GSM and alarm functionality results.

This section focuses on the discussion and analyses of the results obtained during the experiments performed. The experiments performed were to verify if the implemented design was operating properly, hence the results were recorded. These results obtained will be shown in this section and they will be discussed with a support of the theory provided earlier in previous sections. 
Proc. of The Sixth Intl. Conf. On Advances In Computing, Control And Networking - ACCN 2017

Copyright (c) Institute of Research Engineers and Doctors, USA .All rights reserved.

ISBN: 978-1-63248-117-7 doi: 10.15224/ 978-1-63248-117-7-10

\section{A. The results of OOK and FSK}

\section{measured at the receiver at distance of $30 \mathrm{~cm}$}

Figure 5 shows two graphs below are the results for OOK and FSK taken at the distance of $30 \mathrm{~cm}$ following the relevant procedures using oscilloscope. It can be observed that the OOK signal looks clear compared to the FSK signal. The reason could be that the FSK circuit is composed of many components as compared to OOK circuit (check Developments section). Another reason could be the interference of the indoor light rays interfering with the actual information transmitted.

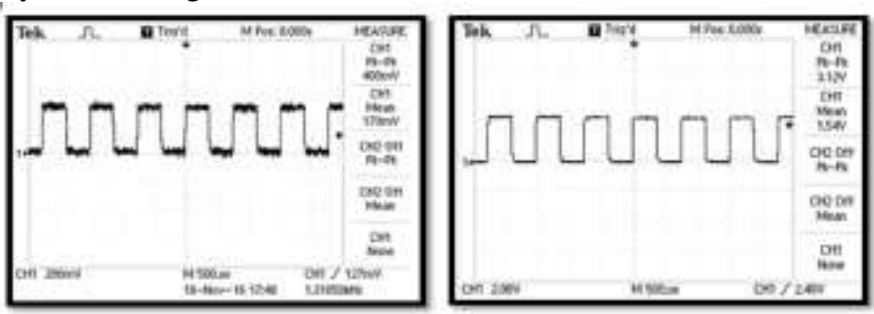

Figure 5: FSK and OOK modulation signals at distance $30 \mathrm{~cm}$

\section{B. The results of OOK and FSK bit error rate versus the distance}

These results recorded below in a table shows that for both OOK and FSK as the distance increase between the transmitter and the receiver circuits the more the errors occur. This makes sense because if the transmitter and the receiver circuits are far apart, then there is much noise interfering with the information transmitted. Moreover distance can affect the sensitivity of the receiver circuit. From the results recorded below it is observed that for $\mathrm{OOK}$ at distance $30 \mathrm{~cm}$ no errors were observed. Therefore, errors started to be detected at $32 \mathrm{~cm}$. For FSK errors were detected from distance $34 \mathrm{~cm}$ to $54 \mathrm{~cm}$.

The 16 bits were sent as to perform the bit error rate in respect to the distance. The massage sent is indicated below, and the right code was used for this. Table 1 shows the equivalent results recorded.

\begin{tabular}{|l|l|l|}
\hline Massage & 0101011001010110 \\
\hline
\end{tabular}

TABLE I: Table of BER versus distance for OOK and FSK

\begin{tabular}{|c|c|c|c|c|}
\hline $\begin{array}{c}\text { Distance } \\
(\mathrm{cm})\end{array}$ & $\begin{array}{c}\text { Number } \\
\text { of errors } \\
(\mathrm{FSK})\end{array}$ & $\begin{array}{c}\text { Bit error } \\
\text { rate (FSK) }\end{array}$ & $\begin{array}{c}\text { Number of } \\
\text { errors } \\
(\text { OOK })\end{array}$ & $\begin{array}{c}\text { Bit } \\
\text { error } \\
\text { rate } \\
(\mathrm{OOK})\end{array}$ \\
\hline 30 & & & & \\
\hline 32 & 1 & -24.08 & 0 & \\
\hline 34 & 2 & -18.06 & 1 & -24.08 \\
\hline 36 & 3 & -14.53 & 2 & -18.03 \\
\hline 38 & 3 & -14.53 & 2 & -18 \\
\hline 40 & 4 & -12.04 & 3 & -14.53 \\
\hline 42 & 5 & -10.01 & 4 & -12.04 \\
\hline 44 & 6 & -8.52 & 5 & -10.1 \\
\hline 46 & 7 & -7.18 & 6 & -8.52 \\
\hline 48 & 7 & -7.18 & 7 & -7.52 \\
\hline 50 & 7 & -7.18 & 7 & -7.52 \\
\hline 52 & 7 & -7.18 & 7 & -7.52 \\
\hline 54 & - & - & 7 & -7.52 \\
\hline
\end{tabular}

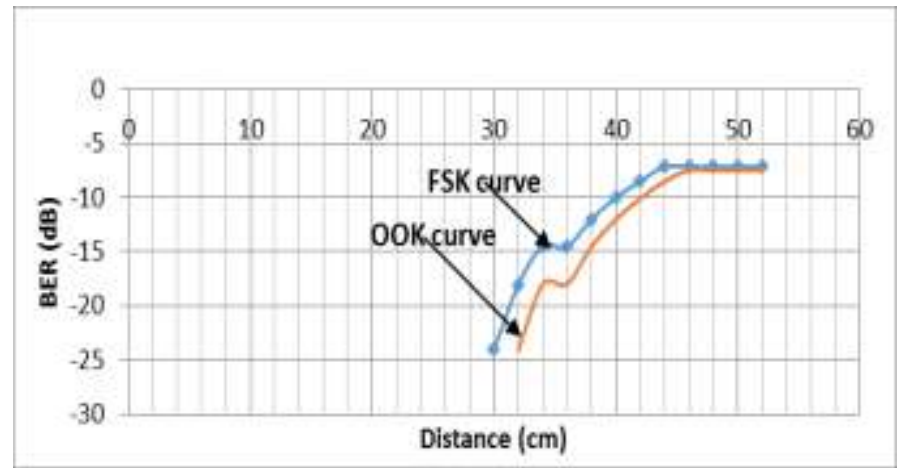

Figure 6: The bit Error rate versus distance curves for OOK/FSK

\section{Conclusion}

The VLC technology is already in a developing stage and is good wireless communication over the existing RF wireless communication. The conduction of this project plays a very big role because already the OOK modulation has standardized for transmission of data in VLC technology. After obtaining the results on this project, it was observed that OOK outperformed the FSK modulation in terms of cost, bit error rate and the simplicity of the circuit.

This actually shows that by making use of OOK modulation in VLC one can save money and can transmit information efficiently. Therefore this shows that it is very important to make use of the best modulation in wireless communication. It is recommended to compare other modulation schemes with OOK modulation, to see if there will perform better. This in return will be beneficial. 
It was observed from the results obtained that the BER increases significantly for all the modulation methods. From circuit affected the sensitivity on the receiver side. This means that there is much interference due to electronic components, which is electromagnetic interference. In addition, the availability of the background noise of the light already existing inside the room also interferes with the information transmitted.

The complexity of the FSK circuit affected the sensitivity on the receiver side. This means that there is much interference due to electronic components, which is electromagnetic interference. In addition, the availability of the background noise of the light already existing inside the room also interferes with the information transmitted.

\section{References}

[1] J. J.George, N. M. Osman, M. H. Mustafa, D. M. Hamed and N. H. Ahmed, "A survey on Visble Light Communication," International journal of engineering and computer science, no. 2, pp. 3805-3808, 2014.

[2] A. Choubey1, "A Comparative study of single-carrier and multi-carrier modulations for visible light communication," Johannesburg, 2013.
Figure 6, it can be seen that the FSK curve approaches the BER at early distance and therefore the OOK performs better than FSK in terms of BER curves. The complexity of the FSK

[3] M. Mutthamma, "A Survey on Transmission of Data through Illumination- Li-Fi," Internation Journal of Research in Computer and Communication Technology, vol. 2, no. 12, p. 1427, December 2013

[4] C. Medina, M. Zambrano and K. Navarro, "LED BASED VISIBLE LIGHT COMMUNICATION:TECHNOLOGY, APPLICATION AND CHALLENGES -A SURVEY," International Journal of Advances in Engineering \& Technology, vol. 8, no. 4, pp. 482-495, 2015.

[5] A. Ndjiongue, H.C.Fereeira and R. Ngatched, "Vissible light Communication (VLC) Technology," Department of Electrical and Electronic Engineering, Johannesburg, 2010.

[6] M. Uysal and M. Farshad, "Channel Modeling and Characterization for visible light communications," IEEE Photonica Journal, vol. Volome 7, no. 6, 2015 .

[7] A. R. NDJIONGUE, HENDRIK C. FERREIRA and TELEX M. N. NGATCHED, "VISIBLE LIGHT COMMUNICATIONS (VLC)," Johannesburg, 2015

[8] "EXAR Monolithic Function generator," EXAR, [Online]. Available: http://www.datasheetarchive.de/EXAR+Monolithic+Function+generator -datasheet.html. [Accessed 2016].

Khmaies Ouahada:

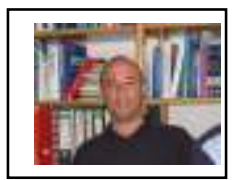

Prof Ouahada is currently lecturing at the University of Johannesburg, South Africa, where he received his M. Eng., with distinction, and his D. Eng. Degrees in 2002 and 2009 respectively. He has received his B. Eng. Degree in 1995 from the University of Khartoum in Sudan. He has worked for a year at Sudatel, the Sudanese National Communications company. He has been employed as a lecturer at the University of Johannesburg in 2008 until 2010 when he was promoted to senior lecturer. In 2013 he was promoted to associate professor. Since his appointment at University of Johannesburg he has lectured Signals and Systems (SST3A11), Telecommunications (TEL3B01) and Digital Signal Processing (SIG3B01) courses for Undergraduates. He is an active member in the Telecommunications Research Group (TRG) and Co-founder of the Wireless laboratory, YLab, at the University of Johannesburg. 